\title{
Using the Correlation Function in Ultrasonic Non-destructive Testing
}

M. Kreidl, P. Houfek

This paper deals with ultrasonic signal de-noising by means of correlation. It is commonly known that the cross-correlation function shows the statistical dependence between two signals. In ultrasonic inspection, the measured signal is taken as the first signal. The most important aspect of this method is the choice of the second signal. Various types of the second signals can be tried.

Keywords: ultrasonic testing, flaw echo, signal noise reduction, cross-correlation.

\section{Introduction}

Ultrasonic testing is a widely used non-destructive testing method for inspection and monitoring. Ultrasonics can be used in many ways for testing. The basic characteristics of an ultrasonic instrument and probe are their sensitivity and resolution [2]. Evaluation of flaws is based on deciding whether a reflector is or is not the flaw echo, and consequently noise reduction is a method that raises the quality of test equipment [3]. Signal de-noising is a critical property of any ultrasonic test system.

\section{Theoretical}

This paper describes the algorithm for noise suppression using the cross-correlation function. The algorithm is based on a well-known equation, Eq. (1).

$$
\hat{R}_{\mathrm{xy}}\left(r T_{\mathrm{v}}\right)=\frac{1}{N} \sum_{n=1}^{N} x\left(n T_{\mathrm{v}}\right) y\left(n T_{\mathrm{v}}+r T_{\mathrm{v}}\right),
$$

where $T_{\mathrm{v}} \quad$ sampling period,

$x\left(n T_{\mathrm{v}}\right)$ ultrasonic signal,

$y\left(n T_{\mathrm{v}}\right)$ simulated signal of the ultrasonic impulse,

$$
y\left(n T_{\mathrm{v}}\right)=0 \text { for } n T_{\mathrm{v}}<0,
$$

$N$ number of samples,

$r=-N, \ldots, N$.

The correlation function can reach the maximum for signals of similar shape. When we measure an ultrasonic signal $x\left(n T_{\mathrm{v}}\right)$ with additive noise, we can use a suitable simulated signal $y\left(n T_{\mathrm{v}}\right)$ for contrasting the flaw echoes. This paper discusses two possible shape models, i.e., two possible simulated signals $y\left(n T_{\mathrm{v}}\right)$ of the ultrasonic impulse with the varying parameters. The parameters should be chosen according to an analysis of the ultrasonic signal with flaw echo.

The first computer simulated ultrasonic impulse signal is expressed by the equation

$$
y\left(n T_{\mathrm{v}}\right)=\mid \begin{array}{cl}
1, & 0 \leq n T_{\mathrm{v}}<T / 2 \\
-1, & T / 2 \leq n T_{\mathrm{V}} \leq T \\
0, & n T_{\mathrm{v}}>T
\end{array}
$$

The model given above represents two rectangular impulses with wide $T / 2$. The second pulse is shifted in relation to the first about the value $T / 2$. Assuming that the reflected ultrasonic impulse has the shape of a damped sinusoidal curve, then signal $y\left(n T_{\mathrm{v}}\right)$ is a sign function of one period of the reflected ultrasonic impulse, and $T / 2$ is a parameter.

For the second simulated ultrasonic impulse signal $y\left(n T_{\mathrm{v}}\right)$, we selected a modulated sinusoidal Gaussian pulse that is very similar to the ultrasonic pulses reflected from the material.

The modulated sinusoidal Gaussian pulse is defined by the following equation

$$
y\left(n T_{\mathrm{v}}\right)=\cos \left(2 \pi f n T_{\mathrm{v}}-\varphi_{0}\right) \cdot e^{-\pi\left(\sigma n T_{\mathrm{v}}-\varphi_{0}\right)^{2}},
$$

where $\varphi_{0} \quad$ phase shift,

$$
\begin{array}{ll}
f & \text { frequency, } \\
\sigma & \text { damping coefficient. }
\end{array}
$$

The phase shift $\varphi_{0}$ can be used for setting the initial phase of the impulse. In our case the value $\varphi_{0}=7 \cdot \pi / 2$ was chosen.

Two variable parameters can be used to achieve conformity with the reflected ultrasonic impulse:

- the frequency of impulse $f$,

- the damping coefficient $\sigma$.

In order to verify the algorithms, the signal $x\left(n T_{\mathrm{v}}\right)$ was simulated in the Matlab environment [1]. This signal simulates a classical high frequency ultrasonic signal with the initial, flaw and final echo without any noise. (Fig. 1).



Fig. 1: Synthetic signal $x\left(n T_{\mathrm{v}}\right)$

The parameters of this synthetic signal correspond to the signal on the output of the ultrasonic device with the working frequency of the acoustic wave equal to $20 \mathrm{MHz}$ and the equivalent sampling frequency equal to $256 \mathrm{MHz}$. The simulated flaw is situated approximately in the middle of a material $10 \mathrm{~mm}$ in thickness. From the statistical analysis of the real ultrasonic noise it is evident that the probability distribution is nonstandard. Therefore the simulation of the ultrasonic noise signal was performed by numerical methods, applying the statistical characteristics of the real noise [1]. 
The variable value of the signal to noise ratio SNR was obtained through increasing and adding to the synthetic signal. The signal to noise ratio SNR can be expressed by Eq. (4)

$$
\mathrm{SNR}=20 \log \left(\frac{U_{\mathrm{ef}}}{N_{\mathrm{ef}}}\right),
$$

where $U_{\text {ef }} \quad$ RMS (root mean square) value of a noiseless ultrasonic signal,

$N_{\text {ef }} \quad$ RMS value of additive noise.

For verification purposes, an analysis was made through the computer-simulated noise signal, corresponding to the value of the SNR coefficient in the range from $0 \mathrm{~dB}$ to $21 \mathrm{~dB}$.

To compare the algorithms, the coefficient of the noise reduction $\delta \mathrm{SN}$ was defined, as follows Eq. (5).

$$
\delta \mathrm{SN}=20 \log \left(\frac{U_{\mathrm{ef} 2}}{N_{\mathrm{ef} 2}} / \frac{U_{\mathrm{ef} 1}}{N_{\mathrm{ef} 1}}\right) \quad[\mathrm{dB}],
$$

where $N_{\text {ef1 }}$ RMS value of the additive noise in the signal,

$N_{\text {ef2 }} \quad$ RMS value of noise included in the signal after application in a given algorithm,

$U_{\text {ef1 }} \quad$ RMS value of the ultrasonic signal, e.g., corresponding to the flaw echo in the measured signal,

$U_{\text {ef2 }} \quad$ RMS value of the ultrasonic signal, when applying the tested algorithm.

The RMS values of the simulated signal were obtained from digital samples corresponding to the flaw echoes before and after application of a given algorithm.

All the above items are non-dimensional:

$$
\begin{array}{ll}
U_{\mathrm{ef} 1}=\left(\frac{u_{1}}{U_{1 \mathrm{max}}}\right)_{\mathrm{ef}} \quad U_{\mathrm{ef} 2}=\left(\frac{\hat{R}_{x y}}{\hat{R}_{x y \text { max }}}\right)_{\mathrm{ef}} \\
N_{\mathrm{ef} 1}=\left(\frac{N_{1}}{N_{1 \text { max }}}\right)_{\mathrm{ef}} \quad N_{\mathrm{ef} 2}=\left(\frac{N_{2}}{N_{2 \max }}\right)_{\mathrm{ef}}
\end{array}
$$

First, the optimum choice of value $T$ was given by correlation analysis between the synthetic signal $x\left(n T_{\mathrm{v}}\right)$ and the simulated ultrasonic impulse $y\left(n T_{\mathrm{v}}\right)$, according to Eq. (2). It is

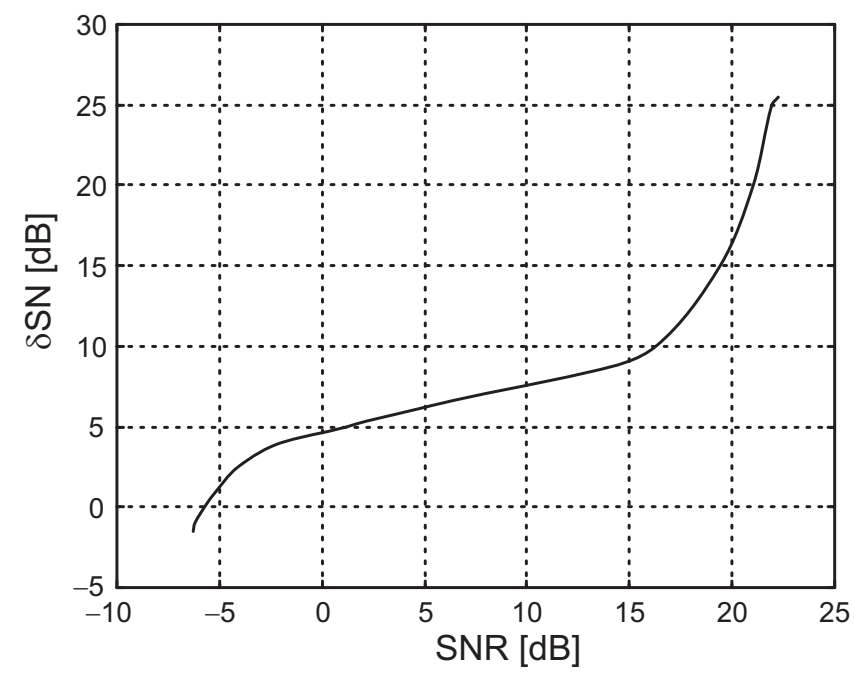

Fig. 2: Dependence of the noise reduction $\delta \mathrm{SN}$ coefficient on the value of coefficient SNR for the optimum value $T=0,05 \mu$ s. The simulated signal was defined by Eq. (2). obvious that value $T$ is determined as the reciprocal value of the acoustic wave frequency. For a frequency of $20 \mathrm{MHz}$ it is consequently $T=0,05 \mu \mathrm{s}$. Then, numerical calculation determined the increase in coefficient $\delta \mathrm{SN}$ depending on value of coefficient SNR. The result is shown in Fig. 2.

Second, the optimum choice of the value parameters according to Eq. (3) was tested. Fig. 3 displays a 3D chart of the coefficient of the noise reduction $\delta \mathrm{SN}$ depending on the acoustic wave frequency and on the Gaussian pulse damping coefficient. The noise level was chosen for the value $\mathrm{SNR}=21 \mathrm{~dB}$. Fig. 3 shows that the maximum noise reduction of the simulated signal $x\left(n T_{\mathrm{v}}\right)$ was achieved with a frequency $f=20 \mathrm{MHz}$ and a damping coefficient $\sigma=9$.

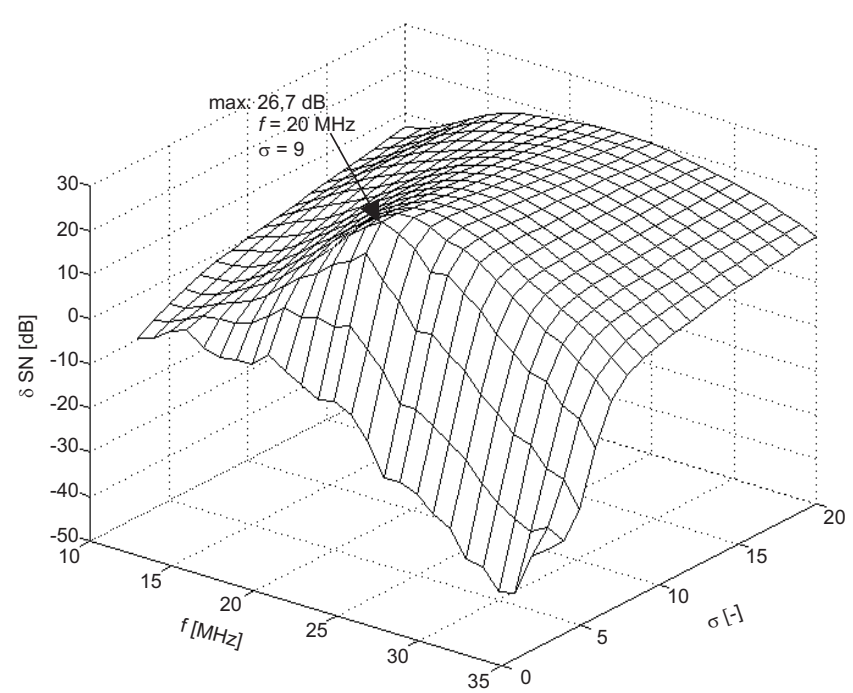

Fig. 3: Relationship between noise reduction coefficient $\delta \mathrm{SN}$ of the synthetic signal $x\left(n T_{\mathrm{v}}\right)$ with the noise ratio $\mathrm{SNR}=21 \mathrm{~dB}$, frequencies $f$ and damping coefficients $\sigma$ of the simulated ultrasonic impulse defined by Eq. (3)

To determine the noise reduction range, the $\delta \mathrm{SN}$ values are displayed in Fig. 4 for different values of the simulated noise levels.

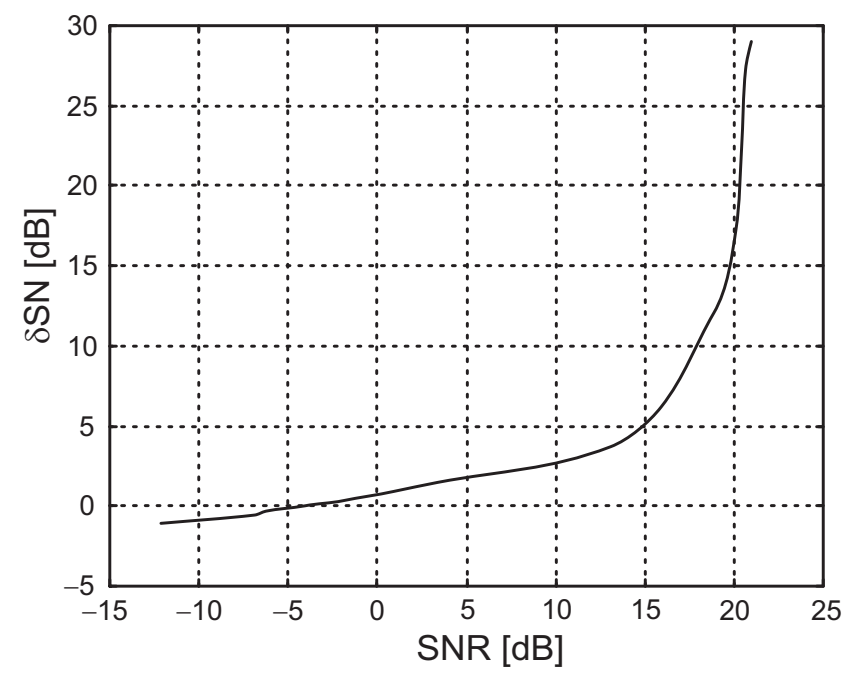

Fig. 4: Dependence of the noise reduction $\delta \mathrm{SN}$ coefficient on the value of coefficient SNR for optimum values of parameters $f=20 \mathrm{MHz}$ and $\sigma=9$ 
As mentioned previously, the results show that this method reduces the noise, owing to the optimum choice of the simulated signal parameters. The suitability of the algorithms is conditioned by the agreement between the flaw wave shape of the real and simulated ultrasonic signals. The ability of the method to reduce noise was then tested on real data.

\section{Experimental}

The proposed noise reduction algorithms, based on the cross-correlation function, were tested on real data [1]. The measurement was performed with the use of a special scale, made of two welded metal sheets $9.2 \mathrm{~mm}$ in thickness. The flaw was artificially manufactured on one part of the scale. Spark technology was used to make a hole $0.5 \mathrm{~mm}$ in dia-

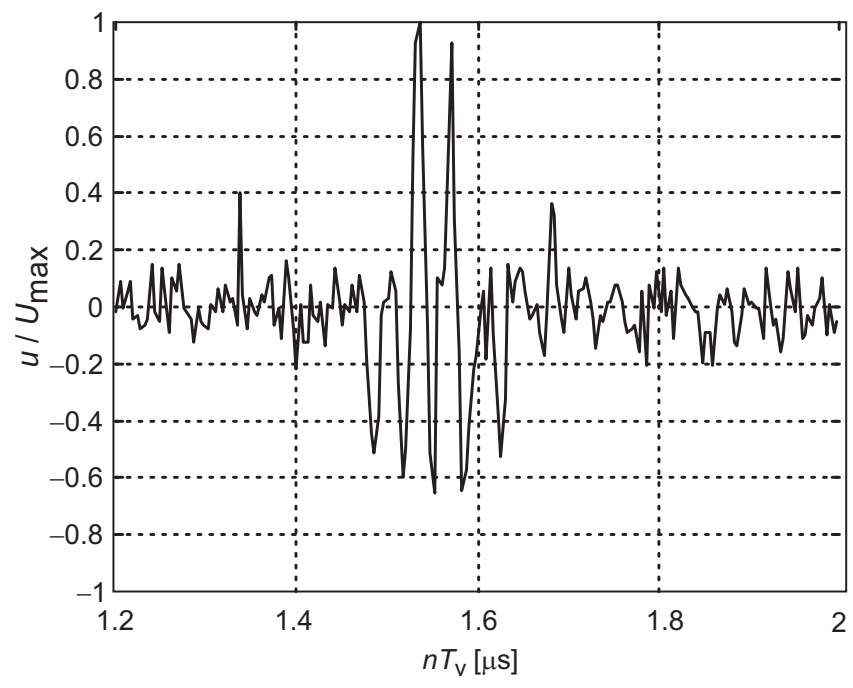

a)



b)

Fig. 5: Results of noise reduction with the use of computation of the cross-correlation function for a simulated impulse, according to Eq. (2) with $T=0.05 \mu \mathrm{s}$ : a) detail of the real signal with flaw echo, b) estimation of the cross-correlation function $\hat{R}_{x y}$. meter. Firstly, in order to test the algorithms, we used the model of the ultrasonic impulse expressed by Eq. (2).

Fig. 5 illustrates signal de-noising with the exception a frequency of $20 \mathrm{MHz}$. This part of the real signal is caused by the reflection of ultrasonic waves from the structure grain boundaries and/or microscopic reflectors in the material.

Next, a simulated signal modulated Gaussian pulse was used, according to Eq. (3). The optimum parameters of this impulse were computed. The change of the ratio $\delta \mathrm{SN}$ in dependence on the parameters is shown in Fig. 6 and in Fig. 7. The values of the noise are drawn without units, because the determination of the noise level is inaccurate. It is evident from the graphs in Fig. 6 and in Fig. 7 that the optimum parameters of the simulated signal are $f=20 \mathrm{MHz}$ and $\sigma=20$.

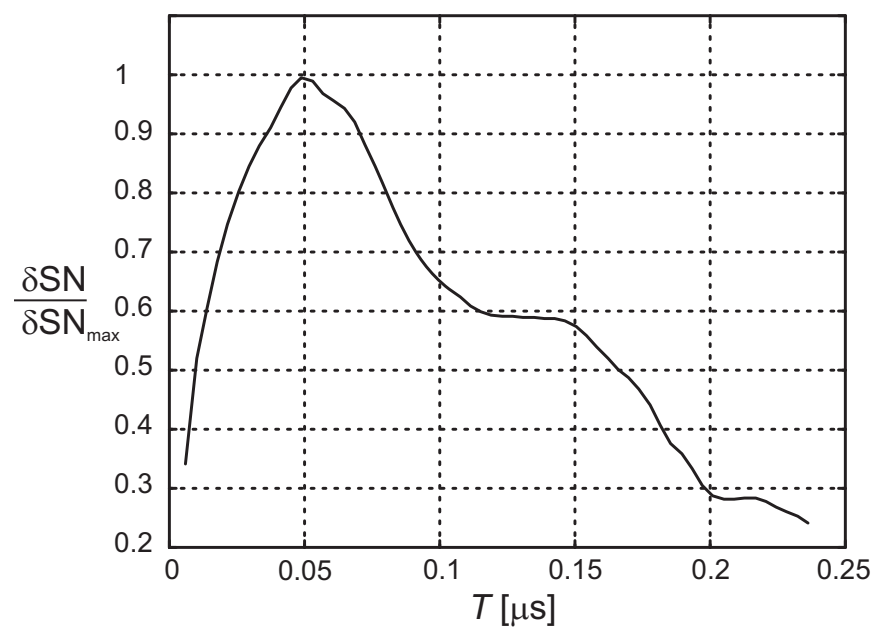

Fig . 6: Dependence of the noise reduction $\delta \mathrm{SN}$ coefficient on different values of $T$ for the simulated ultrasonic impulse, according to Eq. (2)



Fig. 7: Dependence of the noise reduction $\delta \mathrm{SN}$ coefficient on different frequencies $f$ and damping coefficients $\sigma$ for the simulated ultrasonic impulse, according to Eq.(3)

The following figures demonstrate the real ultrasonic signal and this signal after applying the algorithm based on the cross-correlation function with optimum parameters of the Gaussian pulse. 



Fig. 8: Results of noise reduction, using computation of the cross-correlation function for the simulated impulse, according to Eq. (3) with parameters $f=20 \mathrm{MHz}$ and $\sigma=20$

\section{Conclusion}

One of the most important properties of ultrasonic measurement is the suppression of additive noise. Undesirable signal noises arise from contact between the probe and the material, from the amplifier and as a result of scattering at inhomogeneties in the material structure. This paper has provided two algorithms for noise reduction in an ultrasonic signal based on the cross-correlation function. The use of two models of the ultrasonic impulse, according to Eq. (2) and Eq. (3), assuming of the correct choice of model parameters, indicates that the new algorithms are suitable for all practical purposes.

\section{Acknowledgement}

This research work has received support from research program No. J04/98:210000015 Research of New Methods for Physical Quantities Measurement and Their Application in Instrumentation of the Czech Technical University in Prague (sponsored by the Ministry of Education, Youth and Sports of the Czech Republic).

\section{References}

[1] Houfek, P.: Metody zvyšováni citlivosti a rozlišovaci schopnosti v ultrazvukové defektoskopii. [Methods for improving sensitivity and recognition in ultrasonic defectoscopy], Ph.D. thesis. Praha: ČVUT, Fakulta elektrotechnická, 2001.

[2] Kreidl, M., et al: Diagnostické systémy. [Diagnostic Systems]. Monografie, Praha: Vydavatelství ČVUT, 2001, p. 212-228.

[3] Kreidl, M., Houfek, P.: Reducing Ultrasonic Signal noise by Algorithms based on Wavelet Thresholding. Acta Polytechnica, Journal of Advanced Engineering, 2002, Vol. 42, No. 2, p. 60-65.

Doc. Ing. Marcel Kreidl, CSc.

phone: +420224352346

e-mail:kreidl@fel.cvut.cz

Ing. Petr Houfek, Ph.D.

phone: +420224352189

e-mail: houfek@fel.cvut.cz

Department of Measurement

Czech Technical University in Prague

Faculty of Electrical Engineering

Technická 2

16627 Praha 6, Czech Republic 